\title{
Ordinal Linguistic Personification as a Variant of Synesthesia
}

\author{
Julia Simner and Emma Holenstein
}

\begin{abstract}
This study examines the principles underlying ordinal linguistic personification (OLP): the involuntary and automatic tendency in certain individuals to attribute animate-like qualities such as personality and gender to sequential linguistic units (e.g., letters, numerals, days, months). This article aims to provide four types of evidence that OLP constitutes a form of synesthesia and is likely to have the same neurodevelopmental basis. We show that (a) OLP significantly co-occurs with other variants of synesthesia, (b) OLP associations (like those of synesthesia) are highly consistent over time (Experiment 1),
\end{abstract}

\section{INTRODUCTION}

Synesthesia is a familial condition (e.g., Ward \& Simner, 2005; Baron-Cohen, Burt, Smith-Laittan, Harrison, \& Bolton, 1996) in which perceptual experiences (e.g., tastes, sounds) or concepts (e.g., numbers, words) trigger exceptional and incongruous experiences (e.g., colors). The stimulus that triggers the synesthesia is termed the inducer, and the resultant synesthetic experience is called the concurrent (Grossenbacher \& Lovelace, 2001; Grossenbacher, 1997). Synesthetic concurrents can be phenomenologically similar to veridical perception in quality, for example, when colors are seen as externally projected photisms (Ward, Huckstep, \& Tsakanikos, 2006; Simner et al., 2005), and this is known as "projector" synesthesia (Dixon, Smilek, \& Merikle, 2004). In other cases, however, the concurrent equates to a "mind's eye impression" or an overwhelming cognitive association (e.g., the notion of a color; Simner, Glover, \& Mowat, 2006), and this is known as "associator" synesthesia (Dixon et al., 2004).

For both associator and projector variants, the genuineness of synesthesia has been illustrated by behavioral tasks and neuroimaging data. The former show, for example, that the reports of (projector and associator) synesthetes are highly consistent over considerable time intervals, and significantly more consistent than those of matched controls relying on rote memory alone (Rich, Bradshaw, \& Mattingley, 2005; Ward \& Simner 2003,

University of Edinburgh, UK (c) OLP associations (like those of synesthesia) have the characteristic of letter-to-word transference (i.e., they spread from initial letters throughout words) (Experiment 2), and (d) OLP associations (like those of synesthesia) are automatically generated and interfere in Stroop-type tasks (Experiment 3). We argue that these shared characteristics suggest a unified underlying behavior, and propose OLP as a subtype of synesthesia. In so doing, our study extends the range of reported phenomena that are known to be susceptible to cross-modal association.
2005; Palmeri, Blake, Marois, Flanery, \& Whetsell, 2002; Baron-Cohen et al., 1996). Imaging studies have illustrated that associated and projected synesthetic colors (e.g., from grapheme-color synesthesia) produce functional magnetic resonance imaging activation in areas normally associated with the color perception of external stimuli (e.g., V4/V8; Hubbard, Arman, Ramachandran, \& Boynton, 2005; Nunn et al., 2002). The condition is thought to arise from a neurodevelopmental tendency to preserve or develop atypical interactions between brain regions that normally do not communicate (see Hubbard \& Ramachandran, 2005, for a review). Genetic inheritance is assumed to give rise to these atypical pathways independently of the manifested phenotype (Ward \& Simner, 2005; Ward, Simner, \& Auyeung, 2005). Hence, mothers with synesthetic color, for example, can bear children with synesthetic taste, and in this way, all forms of the condition are assumed to stem from the same underlying predisposition for cross talk. The cross talk account has been strengthened by imaging data for synesthetes showing activation of the type predicted (e.g., within adjacent regions of the fusiform gyrus involved in color processing and grapheme recognition; Hubbard et al., 2005).

In this article we describe a possible variant of associator synesthesia, which is the involuntary and automatic tendency in certain individuals to attribute animate-like qualities such as personality and gender to sequential linguistic units (e.g., letters, numerals, days, months). We introduce the term ordinal linguistic personification (OLP) to describe this phenomenon 
and argue that OLP represents a variant of synesthesia by showing that it co-occurs with other variants of synesthesia and shares many of their crucial characteristics. By illustrating equivalence between OLP and synesthesia more generally, we suggest the former is a manifestation of the latter, and hence has the same underlying neurodevelopmental roots. Below we present a review of previous reports of OLPs from the historical and recent literature. We then summarize the phenomenology of OLP after a brief case history of participant A.P., whose OLP experiences will be probed by a subset of our studies. Finally, we present four investigations examining the nature of the OLP experience.

\section{Historical and Contemporary Treatments of OLP}

The phenomenon we have termed OLP has been described in the historical literature, but has yet to be treated in depth in any contemporary peer-reviewed work or to be widely recognized elsewhere in the current literature. Calkins (1893) presented case study reports from individuals experiencing OLP, as well as grapheme-color synesthesia and visuospatial numberslines (i.e., a variant of synesthesia where ordinal sequences such as numbers, letters, days, etc. are experienced in specific spatial arrays; Sagiv, Simner, Collins, Butterworth, \& Ward, 2006). She describes, for instance, the case of a person for whom " $T$ 's are generally crabbed, ungenerous creatures. $U$ is a soulless sort of thing. 4 is honest, but . . 3 I cannot trust. . 9 is dark, a gentleman, tall and graceful, but politic under his suavity" (Calkins, 1893, p. 454). Flournoy (1893) describes a remarkably similar phenomenon, and devotes an entire chapter to this type of experience. The most notable of his case studies is Mme. L., for whom letters and numbers have not only complex personalities, but also relationships of family or association with other units in the series. Her description is translated below (from the original French):

\section{1, 2, 3 are children [who] play together. 4 is a good peaceful woman, absorbed by down-to-earth occupations ... 5 is a young man, ordinary and common in his tastes and appearance, but extravagant and self-centered. 6 is a young man ... polite, gentle, ... average intelligence; orphan. 7 is a bad sort, although brought up well; spiritual, extravagant, gay, likeable; capable of very good actions on occasion ... 8 is a very dignified lady, who acts appropriately... She is the wife of 9 [who is] self-centered, maniacal, grumpy, endlessly reproaching his wife for one thing or another. (Flournoy, 1893, pp. 219-220)}

Patrick (1893) describes two additional cases, which are similar in their complexity both to each other and to Mme L. For example, for one participant, the number 5 is "a society girl ... [who] has everything she wants; does not care how much trouble she makes other people," whereas for the other, it is "a business or professional man, successful and not particularly intellectual" (Patrick, 1893, p. 509). Both cases were female, and neither person could provide any explanation for the causes or origins of their associations.

There are, to our knowledge, only three mentions of OLP in the current literature, which describe the phenomenon as a minor focus, and provide only partial claims about its status. Cytowic (2002) describes participant M.T., who experiences letters and numbers with colored photisms, as well as human physical and psychological attributes. For example, the letter $d$ is a charcoal gray color, and "male, dashing, a bit of a joker" (Cytowic, 2002, p. 298). It is not clear whether Cytowic considers OLP as a manifestation of synesthesia in its own right, or simply as the collateral experiences of a grapheme-color synesthete. Sagiv (2005) briefly describes the phenomenon as a synesthetic variant, but without discussion of this assumption. Finally, Simner, Mulvenna, et al. (2006) also suggest that OLP may be a form of synesthesia, but exclude it from their estimate of synesthesia's prevalence, because the contemporary literature had yet to provide a definitive classification of the phenomenon.

From these reports, it is possible to draw tentative conclusions about the particular characteristics of OLP, such as the range of possible inducers and concurrents, and their qualitative natures. First, however, we introduce participant A.P., a focus of the current study, because her case will allow us to present a more complete phenomenology.

\section{Case Study A.P.}

A.P. is a 23-year-old right-handed female graduate, who reports OLP as well as a number of additional synesthetic phenomena. Her letters, numbers, and days trigger automatic, consistent, and specific attributes of color, and she experiences the impression of tactile shapes in response to tastes. These forms of synesthesia (grapheme-color, day-color, taste-shape, respectively) have been independently verified elsewhere with objective tests of genuineness (Simner, Mulvenna, et al., 2006). Additionally, she reports colors for some words, as well as shape concurrents triggered by pain, and a visuospatial number-line.

The OLP experiences of A.P. are triggered by three types of linguistic ordinal sequence: Letters, numerals, and months, and examples of these associations are shown in Table 1 (a full list is available from the first author; see also Simner \& Hubbard, 2006). Her OLP concurrents are highly detailed, and A.P. goes to considerable trouble to describe them. This characteristic has been seen in previous reports of OLP and has been observed in other areas of synesthesia more generally (e.g., Simner et al., 2005; Ward \& Simner, 2003). A.P. 
Table 1. Examples of OLP Inducer-concurrent Associations for Participant A.P.

\begin{tabular}{ll}
\hline Inducer & \multicolumn{1}{c}{ Concurrent Personifications } \\
\hline 1 & m: "a good guy"; responsible; father figure; nice; a little tired \\
4 & m: good person; energetic; young; lots of "get-up-and-go"; well-balanced \\
5 & f: mother figure; funny by accident; does things around the house \\
7 & m: submissive, weak man; not very confident \\
8 & f: fat; dating 9 but loves 7 (who'd be a great match but everyone prefers 6 with 7 ) \\
e & m: "a cheeky chappy"; talks when he doesn't know what he's talking about \\
f & m: "a dodgy geezer" (i.e., untrustworthy); has connections; like $e$ but less nice \\
i & m: little guy; $b$ and $g$ are always fussing over him; independent; wants to get on \\
k & f: energetic; bubbles along; not always approachable; mother of shy/quiet son $l$ \\
m & f: old lady like $n$; they spend all their time together and gossip a lot \\
January & f: sensible; not many friends; introvert; if January has a friend it's February \\
February & f: underdog to January; nicer; not so introvert, January has more standing \\
June & f: beautiful, popular; best friends with July; slightly snobby \\
August & m: similar to October; both chubby, boys among girls, and may be defensive \\
December & m: a young guy; really, really nice; protective over the rest; maybe a boss \\
\hline
\end{tabular}

$\mathrm{f}=$ female; $\mathrm{m}=$ male.

reports that she has had her OLP (and other synesthetic) associations for as long as she can remember and is unaware of their origins.

\section{Phenomenology of OLP}

From the case study here and those from Cytowic (2002) and the historical literature, we can draw the following conclusions about the phenomenology of OLP. Attested OLP inducers have been letters, numbers, days, and months, and our terminology reflects the fact that these are linguistic units from ordinal sequences. These inducers give rise to a range of complex concurrents, which we summarize into eight categories. Five of these reflect individual characteristics, and three are relationships between units in the sequence. These characteristics are of gender (e.g., male), personality (e.g., generous), physical appearance (e.g., fat), occupation (e.g., businessman), cognitive attributes (e.g., intelligent), familial relationships (e.g., mother), relations of nonfamilial association (e.g., neighbor, friend), and emotive responses to other units (e.g., X loves/pities/ annoys Y). Finally, our observations lead us to propose two further characteristics. First, it appears that relationships between units tend to hold within, but not across, sequences (e.g., 7 might be the father of 6 but not of the letter $f$ ) and, moreover, only between units that are proximal in a sequence (e.g., $p$ might be the son of $q$, but not of a distal unit like $b$ ). Second, we suggest that concurrents may be influenced by experience, because the personality types tend to reflect the society that is contemporary to the report. Older studies, for example, mention "society girls" and "housekeepers," whereas such descriptions are less apparent in modern accounts. We now examine the nature of OLP in more detail, with four empirical investigations.

\section{EXPERIMENTAL MANIPULATIONS}

The studies here are designed to provide evidence that OLP is a variant of synesthesia by showing that it significantly co-occurs with other variants and that it shares with them crucial characteristics. The characteristics we test are those of consistency, automaticity, and letter-toword transference (i.e., the tendency to spread concurrent features from a letter to any word for which that letter serves as the initial constituent). These studies are described below.

Experiment 1 tests whether OLP shares with synesthesia the feature of consistency over time. This is a fundamental characteristic of synesthesia (Rich et al., 2005; Ward et al., 2005; Ward \& Simner, 2003, 2005; Baron-Cohen et al., 1996; Baron-Cohen, Wyke, \& Binnie, 1987) and the test of consistency has been described as the "behavioral gold standard" for synesthetic genuineness (Rich et al., 2005). In standard tests, inducerconcurrent pairings (e.g., $a=$ red, $b=$ blue) are elicited from the participant in an initial session, and then again in a surprise retest many months or even years later. Synesthetes are significantly more consistent than 
controls, even when these latter are tested over far shorter intervals (e.g., 2 weeks). We use the same methodology to examine whether participant A.P. shows similar high levels of consistency for her OLP associations.

Next, we test whether OLP significantly co-occurs with other variants of synesthesia, because multiple forms of synesthesia are often found to occur within the same individual (e.g., Simner, Mulvenna, et al., 2006; Ward \& Simner, 2005; Flournoy, 1893). Specifically, we test for the co-occurrence OLP with grapheme-color synesthesia by identifying the number of people with one or both conditions within a sampled population. Genuineness will be verified by the behavioral standard of consistency over time (following the outcome of Experiment 1), and we assess whether the two conditions are more likely to cooccur than would be predicted by chance. Any meaningful co-occurrence would suggest that OLP may be one of the range of manifestations of the synesthetic experience.

Experiment 2 tests whether OLP shares with synesthesia the tendency for the initial letter/number in any composite unit to dictate the characteristic of that unit as a whole. For example, the synesthetic color of a word can be inherited from the color of the initial letter (e.g., the word art tends to be the color of the letter $a$; Rich et al., 2005; Ward et al., 2005; Calkins, 1893), and multidigit numbers can take the color of the initial numeral (e.g., for case A in Calkins, 1893, the number 2348 is red because 2 is red; see also Tyler, 2005). We seek to examine whether OLP shares this same characteristic and do so by exploiting the semantic gender inherent in English proper names. Names are selected whose semantic gender is either congruent with participant A.P.'s OLP gender of the initial letter (e.g., Brian, where $b$ is a male letter) or incongruent with it (e.g., Betsy). The participant is asked to rate each name for how masculine or feminine is its referent. If words take on the personification gender of the initial letter, then their lexical-semantic gender should be modified by this characteristic, and names in the incongruent condition should be rated lower on a scale of their true semantic gender (e.g., incongruent girls' names should be rated less feminine).

Experiment 3 tests whether OLP shares with synesthesia the feature of automaticity by examining whether the effects found in Experiment 2 represent automatic processing (rather than conscious off-line strategic effects). In other domains, online Stroop-type experiments (Stroop, 1935) have shown that synesthetic concurrents are automatically generated and can interfere with reaction time (RT) processing if they conflict with the features of the stimulus. For example, responses in font-color naming for colored graphemes are slower if that color conflicts with the synesthetic color of the target (e.g., responding to a green font for letters that are synesthetically red). We again exploit the congruency manipulation with English proper names, but now present an online speeded response task to indicate whether stimuli are boys' names or girls' names. If gender personifications cause Stroop-type interference, we would expect slower responses when real-world gender and OLP gender clash (e.g., Betsy, where $b=$ masculine). From this we could draw two conclusions: First, the gender of letters is elicited automatically, even where this makes the task at hand more difficult. Second, because interference in Stroop-type tasks has been used as evidence of genuineness for other variants of synesthesia (e.g., sound-taste, grapheme-color, e.g., Beeli, Esslen, \& Jäncke, 2005; Mills, Boteler, \& Oliver, 1999) a similar finding here would lend support for the genuineness of OLP.

\section{Experiment 1}

This study tests whether OLP associations are consistent over time and whether they are significantly more consistent than those of a group of matched control participants producing analogous associations from rote memory alone.

\section{Method}

Participants. Forty English-speaking control participants (28 women, 12 men; mean age, 22.6 years) were recruited from the University of Edinburgh community and matched to participant A.P. in approximate age and educational background. A.P. was paid $£ 30$ to participate in this study and Experiments 2 and 3. Control participants were screened to ensure that none experienced OLP or any other synesthetic variant. For screening, participants were given a booklet that described all known variants of synesthesia, and which prompted participants to indicate any variant they thought they may have. Any participant providing any type of affirmative response was excluded from the control group.

Procedure. Control participants were divided into two groups. Half were presented with a booklet listing the letters of the alphabet and numerals up to 10, and the other half were given the months of the year and the days of the week. (The data for days were collected for a study to be reported elsewhere.) A.P. was given the list of letters, numerals, and months, and asked to write her OLP gender and personality for each item, whereas controls were asked to invent analogous associations. Control participants and A.P. then performed a surprise retest after 3 weeks and 2.1 years, respectively. A.P. was required to repeat her personifications, and controls were asked to remember what they had written previously or to guess if they could no longer remember.

\section{Results and Discussion}

The control participants' percentage mean scores for accuracy in gender and personality are shown in Table 2 , 
Table 2. Percent Consistency Scores for A.P. across Three Ordinal Sequences, for Gender and Personality, and Comparable Mean Percent Scores (and Standard Deviations) for Control Participants

\begin{tabular}{lccccc}
\hline & \multicolumn{2}{c}{ Gender } & & \multicolumn{2}{c}{ Personality } \\
\cline { 2 - 3 } \cline { 6 - 6 } & A.P. & Controls & & A.P. & Controls \\
\hline Numerals & 100 & $52.5(19.4)$ & & 100 & $12.6(9.3)$ \\
Letters & 100 & $63.1(11.2)$ & & 100 & $14.4(8.9)$ \\
Months & 100 & $60.8(20.0)$ & & 91.7 & $25.8(17.5)$ \\
\hline
\end{tabular}

along with their standard deviations. Also shown are A.P.'s associations, which were highly consistent over time; indeed, there was only one response that was not identical over her $>2$-year testing period. (This was for March, which was described as a young female on both occasions, but not specifically as wealthy/serious at Time 2).

Despite the considerably longer retest interval, A.P. scored significantly higher than controls for the gender and personality of letters $(Z=3.3, p<.001 ; Z=9.7$, $p<.001)$, numerals $(Z=2.5, p<.02 ; Z=9.7, p<.001)$, and months $(Z=2.0, p<.05 ; Z=3.8, p<.001)$, and from this we draw two conclusions. First, OLP shares with other synesthesias the key characteristic of consistency over time (Rich et al., 2005; Ward \& Simner 2003, 2005; Baron-Cohen et al., 1987, 1996), and second, this consistency - as in other areas of synesthesia-might be taken as evidence of genuineness (e.g., Baron-Cohen et al., 1987).

\section{Do OLP and Grapheme-Color Synesthesia Co-occur?}

Experiment 1 illustrates that OLP inducer-concurrent pairings are significantly consistent over time, and we can now use this characteristic to test an additional hypothesis about the OLP phenomenon. It is known that different variants of synesthesia tend to co-occur (e.g., Simner, Mulvenna, et al., 2006). Therefore, if OLP is indeed a variant of synesthesia, we might expect that it, too, would tend to significantly co-occur with other variants, and to test this, we examined the prevalence patterns of OLP and grapheme-color synesthesia (which we selected because it is a common variant; Simner, Mulvenna, et al., 2006). Specifically, we tested a random sample of 219 members of the University of Edinburgh community (106 men, 113 women; mean age, 22.6 years) for the presence of these two conditions and used consistency over time as an objective test of genuineness (following our findings from Experiment 1). We aimed to show whether OLP and grapheme-color synesthesia are significantly likely to co-occur, and hence, whether they might be different manifestations of the same underlying condition.
Participants completed a questionnaire asking whether they had previously (i.e., before the testing date) believed that letters and/or numbers had particular genders and/or personality traits and/or colors. Participants who reported any type of affirmative response $(n=78)$ were asked to write down their associations for the letters of the alphabet and numerals up to 10 (with the option to leave blank any items that had no association). Participants were then given a surprise retest after 5 weeks, and their consistency scores were compared to two groups of controls, each tested after only 2 to 3 weeks. Scores for gender and/or personality were compared to the control group $(n=20)$ described in Experiment 1, and scores for consistency in color were compared to controls $(n=40)$ from Simner, Mulvenna, et al. (2006). Those participants scoring significantly higher than controls were classified as potential synesthetes and were given an additional surprise retest after 1.3 years. This long delay was to separate those who had performed well at 5 weeks into two subgroups: genuine synesthetes/personifiers (whose associations would endure) versus those who had initially performed well from superior memory alone.

Of those 78 participants who initially reported some type of OLP and/or grapheme-color synesthesia, 21 performed significantly higher than controls at 5 weeks, and 10 of these continued to score significantly higher (than the controls' 2- to 3-week consistency) in the surprise retest over a year later. Of these 10 genuine cases, 7 had grapheme-color synesthesia only, 2 had OLP only, and 1 had both. ${ }^{1}$ If we divide the population of 219 participants into personifiers and nonpersonifiers, 33.3\% of the former had grapheme-color synesthesia, compared with only $3.2 \%$ of the latter. Hence, people with OLP are more likely to have grapheme-color synesthesia than people who do not $\left(\chi^{2}=7.6, p<.01\right)$ suggesting the two conditions are significantly likely to co-occur. This in turn provides support for the notion that OLP is itself a variant of synesthesia, inasmuch as different variants are likely to coexist within the same individuals.

\section{Experiment 2}

The aim of this study was to examine whether OLPs function similarly to synesthesia in the relationship between the attributes of letters and words. In other forms of synesthesia, the concurrent of a letter can spread throughout a word for which that letter serves as the initial constituent (e.g., words tend to take the synesthetic color of the initial letter; Simner, Glover, et al., 2006). In the current study we presented English proper names whose semantic gender was either congruent with the OLP gender of the initial letter (e.g., Brian, where $b$ is a male letter) or incongruent with it (e.g., Betsy). Participant A.P. rated each name for how masculine or feminine she believed its referent to be. If the OLP gender of the initial letter spreads throughout the 
word, we predict that names in the incongruent condition should be rated lower on a scale of their true semantic gender (e.g., incongruent girls' names should be rated less feminine).

\section{Method}

Materials. The materials comprised 112 proper names ( 56 male, 56 female), half in the congruent condition (28 male, 28 female) and half in the incongruent condition (28 male, 28 female). Names were unambiguously male or female (i.e., names like Pat were removed). In the congruent condition the semantic gender of the name matched the OLP gender of the initial letter (e.g., Brian, where $b$ is OLP male), whereas in the incongruent condition, there was a mismatch (e.g., Betsy). Names were balanced pairwise by their initial letter, letter length, syllable length, and lexical frequency (Kucera and Francis; www.psy.uwa.edu.au/MRCDataBase/uwa mrc.htm). Respective means for letters, syllables, and frequency were as follows: congruent $=5.5,1.9,0.3$; incongruent $=5.5,1.9,0.3$.

In the event that OLP gender might generate differential effects for male versus female associations, items were also balanced pairwise across names whose initial letters had male versus female OLP genders. Respective means for letter length, syllable length and frequency were as follows: female OLP $=5.4,1.9,0.3$, male OLP $=$ $5.5,1.9,0.3$.

Procedure. Names were presented in an electronic booklet, in a randomized order. Each name was accompanied by a scale consisting of a line running from "extremely male" (left) to "extremely female" (right) in 22 equal parts, with its midpoint indicated by a double line ("I"). The participant was instructed to rate each name for her subjective impression about the maleness/ femaleness of its referent by placing an " $\mathrm{x}$ " on the scale. The instructions also explained that any response that was perfectly between the two extremes should be placed between the midpoint lines (i.e., "extremely male ------- $|x|----$ extremely female"). The participant was encouraged to ask for clarification, and the procedure took approximately $25 \mathrm{~min}$.

\section{Results}

Responses were coded on a scale from 1 to 7 in 0.25 increments (i.e., $1.00,1.25,1.50,1.75, \ldots$, etc.). In this, each increment corresponded to each gap between the segments making up the line scale (see above). Because responses within each of the congruent and incongruent conditions comprised both male and female names, there could be no consistent mapping between absolute position on the scale from left to right, and a simple increase from 1 to 7 representing the influence of congruency. For this reason, responses were given the highest value (7) if they were placed at the extreme end corresponding to the true semantic gender of the name (i.e., for boys' names, 7 is allocated to an extreme lefthand response, and for girls' names, an extreme righthand response). This manipulation meant that scores now represented the degree to which the rating approached the true semantic gender of the proper name. The resultant mean ratings in each condition are shown in Figure 1.

A repeated measures two-way analysis of variance (ANOVA) revealed a main effect of congruency, $F(1,27)=$ $45.7, p<.001$, but no effect of OLP gender, $F(1,27)<1$, and no interaction, $F(1,27)=1.2, p>.05$. Hence, the personification gender of the initial letter influenced the perceived real-world semantic gender of the proper name, giving rise to a congruency effect (higher scores for congruent items), which is equivalent for OLP male and OLP female letters.

\section{Discussion}

This experiment shows that judgements about the lexical-semantic gender of words are influenced by the OLP gender of the initial letter. Hence, proper names beginning with OLP female letters, for example, are judged to have referents that are more female. From this we conclude that OLP, like other variants of synesthesia (e.g., grapheme-color) has the quality of letter-to-word transference (i.e., the tendency to spread a concurrent feature from a letter throughout any word for which that letter serves as the initial constituent).

As a note, we point out that this characteristic of letter-to-word transference does not extend to month names, whose genders/personalities are independent of those for their composite letters (e.g., February is female, whereas $f$ is male). Indeed, this constitutes further evidence of synesthesia-like mechanisms in OLP, simply because this same month-related exception is often seen in other variants of synesthesia. For example,

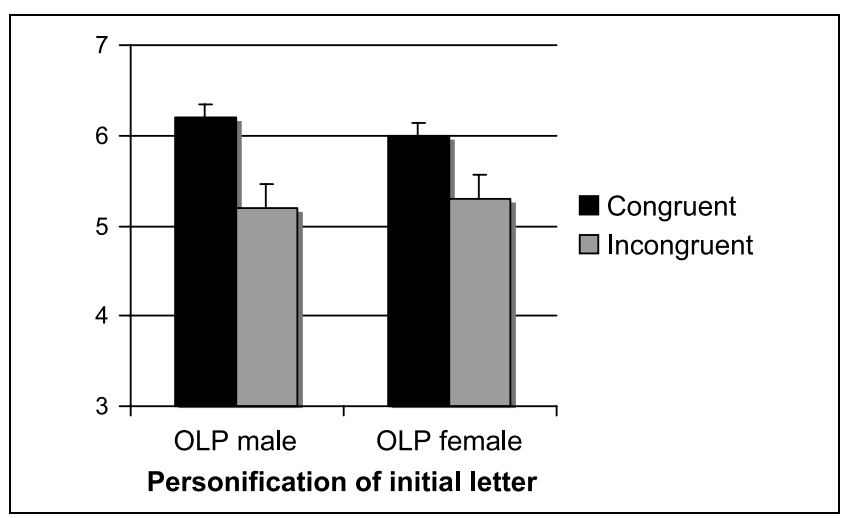

Figure 1. Mean gender ratings of the referent of proper names. Higher ratings $(\max =7)$ correspond to judgments that are closest to the true semantic gender (e.g., 7: John = extremely male; 1 : John $=$ extremely female). 
although words may be colored by their initial letter in grapheme-color synesthesia (e.g., Ward et al., 2005), day names and month names are often the exception to (e.g., although janitor and Jane, etc. might be colored by $j$, the word January would not; Rich et al., 2005). In this way, OLP exhibits not only the synesthetic rule of letter-to-word transference, but also includes the same exceptions to this rule.

\section{Experiment 3}

The aim of Experiment 3 was to examine whether the effects found in Experiment 2 represent automatic processing, or simply conscious off-line strategic effects. In Experiment 2, participant A.P. was allowed to respond at leisure and may have performed according to some type of meta-reasoning not reflective of usual automatic processing. The current experiment uses an online Stroop-type paradigm (Stroop, 1935) with materials similar to those of Experiment 2. A.P. made speeded, objective responses classifying items as either boys' or girls' names, and we predict slower responses in the incongruent condition (e.g., Betsy, where $b=$ masculine). From this we would conclude that the gender of letters is elicited automatically. Additionally, a Stroop-type interference would suggest that OLP reports are genuine (e.g., Beeli et al., 2005; Mills et al., 1999).

\section{Method}

Materials. The materials were similar to Experiment 2, but a greater number were included to improve the estimates of means. They now comprised 188 names (94 male, 94 female), half in the congruent condition ( 47 male, 47 female) and half in the incongruent condition ( 47 male, 47 female). Names were balanced in the way described in Experiment 2, and respective means for the number of letters, number of syllables and frequency across conditions were as follows: congruent $=5.4,1.9$, 3.5 ; incongruent $=5.4,1.9,3.5$; OLP female $=5.4,1.9$, 3.5; OLP male $=5.4,1.9,3.5$.

Procedure. The participant was seated at a computer running E-Prime software (Psychological Software Tools, 2002) with a high-resolution monitor. Each trial began with an on-screen fixation point ("+"). After $1500 \mathrm{msec}$, this was replaced by a proper name, randomly selected without replacement from the materials list. All stimuli were presented in white font 48 Times New Roman against a black background and remained on-screen until the participant made a response from a button box that had two buttons revealed, marked MALE and FEMALE. The participant was required to use the button press to indicate whether the item was a boy's name or a girl's name, and to do so as quickly and accurately as possible. The experiment lasted approximately $10 \mathrm{~min}$.

\section{Results}

There were 20 errors, equally distributed throughout the data. These were removed, and the remaining RTs treated for outliers by removing all data points beyond two standard deviations from the mean. Outliers represented $3.7 \%$ of the data and were equally distributed across conditions. The resultant means across the remaining items are shown in Figure 2.

A repeated measures two-way ANOVA revealed a main effect of congruency, $F(1,46)=21.1, p<.001$, but no effect of OLP gender, $F(1,46)<1$, and no interaction, $F(1,46)=2.0, p>.05$. Hence, the personification gender of the initial letter influenced RTs to the real-world semantic gender of the proper name, giving rise to a congruency effect (faster for congruent items), which is equivalent for OLP male and OLP female letters.

\section{Discussion}

Results show that processing slows when the personification gender of the initial letter clashes with the realworld lexical-semantic gender of the proper name. Given that the task required a nonsubjective response ("Are these boys' or girls' names?") and elicited speeded online response times, our findings suggest that the OLP gender of the initial letter is automatically generated, even where this makes the task more difficult. Finally, as in other variants of synesthesia, we present the Strooptype slowdown as support for the genuineness of OLP.

\section{GENERAL DISCUSSION}

\section{Similarities between OLP and Synesthesia}

To our knowledge, this study represents the first empirical treatment of OLP in the contemporary peerreviewed literature, and we have used our findings to provide four types of evidence that OLP constitutes a form of synesthesia. We show that OLP significantly cooccurs with other variants of synesthesia, and that OLP

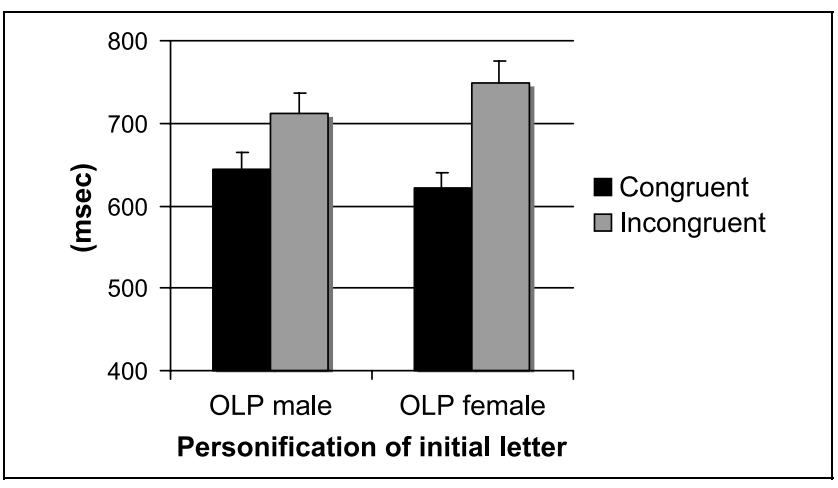

Figure 2. Mean RTs (milliseconds) for the assignment of semantic gender of proper names. 
associations, like those of other synesthesias, are highly stable over time, cause automatic congruency effects in Stroop-type tasks, and transfer their characteristics from a letter throughout a word. In addition to these empirically supported similarities, others emerge from first-person reports: Both synesthetic and OLP concurrents share their intensity and early onset, and both are highly detailed such that participants go to some lengths to describe them (Ward \& Simner, 2003). Furthermore, many variants of synesthesia (82\%; Simner, Mulvenna, et al., 2006) are triggered by ordinal sequences (e.g., grapheme-color, day-color, month-color, numberlines) and the same is true of OLP. Finally, the OLP phenomenon fits with contemporary definitions of synesthesia (e.g., Simner, Mulvenna, et al., 2006; Dixon et al., 2004; Dixon, Smilek, Cudahy, \& Merikle, 2000) even where these have not been constructed to specifically include this phenomenon.

\section{Neurodevelopmental Causes of OLP}

Our findings have provided possible indications about the causes of the OLP experience, and we discuss this now. We begin with the observations that all humans, synesthetic or otherwise, have a tendency to consciously construct personifications, and this is known as anthropomorphism (Morris, Fidler, \& Costall, 2000) or animalism (Looft \& Bartz, 1969; see also Piaget, 1929, 1933). This has been observed in numerous areas, from the personification of animals (Morris et al., 2000) to machines (e.g., computers; Marakas, Johnson, \& Palmer, 2000) to abstract notions such as time and death (Guthrie, 1993), and it is known to extend across cultures (Looft \& Bartz, 1969). This raises the possibility that OLPs may be some pathological or disinhibited form of this common human trait. However, we argue against this for two reasons. First, general attributions of anthropomorphism tend not to extend to linguistic units, although these are the clear triggers in the OLP experience. In other words, the very specific nature of the OLP inducer makes us suspect an unrelated cause. Second, instances of everyday anthropomorphism tend to arise from analogical characteristics in the stimulus (e.g., a computer that has a non-user-friendly interface might be considered "unhelpful"), although there are few obvious humanlike qualities inherent in the linguistic units that trigger OLP (e.g., the letter $e$, the number 2). We suggest then that despite the superficial similarity, OLP may not arise from an extension of the same general processes of anthropomorphic metaphor.

An alternative suggestion, from Flournoy (1893), is that OLP may arise via associations inherent in other forms of synesthesia (e.g., day-color; grapheme-color). Flournoy suggests that synesthetic colors may become elaborated to form the more complex associations of OLP, such that a blue Friday or red Saturday, for example, might become a woman dressed in blue or a man dressed in red. Elsewhere we show that the features of OLP and color synesthesia do indeed interact in cognitive tasks (Simner \& Hubbard, 2006), which may lend support for Flournoy's hypothesis. However, his account predicts that OLP cannot exist in the absence of color synesthesias, although our prevalence survey contradicts this. Moreover, it is not entirely clear how unidimensional features of color could come to be represented as multifaceted characteristics of physical, psychological, moral, familial, or professional qualities. Flournoy suggest this occurs in a conscious processthat OLP is expressly and consciously elaborated in the same way, for example, as childhood imaginary friends (Flournoy, 1893). However, this conflicts with reports throughout the OLP literature where participants readily state that they have no idea about the roots of their associations and no memory of constructing them (and this is true for our participant A.P., as well as for Flournoy's Mme. L.). Finally, given that we have provided empirical evidence for OLP as a variant of synesthesia, it should be no surprise to find that OLP associations are no more consciously constructed than those of the tastes or colors found in lexical-gustatory or grapheme-color synesthesia (e.g., Ward et al., 2005; Ward \& Simner, 2003).

In place of the color to personality bootstrapping account of Flournoy (1893), we propose a more direct cause for OLP, similar to proposals for other variants of synesthesia. Current accounts of synesthesia attribute it with a neurological basis endowed by biological inheritance (Hubbard et al., 2005; Ward \& Simner, 2005; Nunn et al., 2002; Maurer, 1997; Baron-Cohen et al., 1996). In this, a genetic predisposition is assumed to cause cross talk between areas of the brain that would otherwise have no functional association in the normal, nonsynesthetic adult (Maurer \& Mondloch, 2005; Grossenbacher, 1997; Maurer, 1997; Baron-Cohen et al., 1996). The same inherited predisposition is known to give rise to a range of manifested synesthetic phenotypes (Ward \& Simner, 2005; Ward et al., 2005) according to the areas that become functionally associated in the resultant adult structure. The fact that our study has found OLP significantly co-occurring with other synesthesias and sharing their characteristics raises the possibility that OLP may be caused by the same neurodevelopmental mechanism(s). In other words, inherited synesthetic cross talk might associate not only "conventional" inducers and concurrents (e.g., sounds, colors), but also those involved in the OLP experience. As such, OLP might be considered just one of the number of conditions that arises from the inherited synesthetic tendency to preserve or develop atypical projections in development.

This proposal may have some merit given findings from recent imaging studies about the attribution of personality traits. Heberlein and Saxe (2005) examined the neurological activity in normal participants engaged in a task of personality trait attribution to point-light walker 
stimuli (i.e., stimuli created by attaching lights to a body whose movement is filmed in the dark). When participants made personality trait judgements, Heberlein and Saxe found activation in the left inferior frontal gyrus. Crucially, they note that this region has been widely linked to verbal semantic processing (e.g., Chien \& Fiez, 2001; Poldrack et al., 1999) and suggest that knowledge about personality traits may be encoded in regions closely associated to those encoding linguistic knowledge. Ramachandran and Hubbard (2001a, 2001b) have suggested that synesthetic cross-talk may be especially encouraged between areas that are geographically local. It is reasonable to suggest, therefore, that cross-talk between personality trait and language areas may indeed be at the root of OLP associations. This hypothesis is appealing (see also Simner \& Hubbard, 2006) but remains tentative until neuroimaging studies provide information about the precise activity generated by OLP processing.

\section{Influences of Learning and Experience}

Notwithstanding this neurodevelopmental account, we suggested that OLP can also be influenced by experience. At a very fundamental level, OLPs must necessarily be modified by learning because their inducers are learned linguistic units (letters, numbers, words). In addition, however, we might also see an influence of learning in the nature of the concurrent. We noted above that certain OLP personality types appear to reflect the era of the report (e.g., society girls in the early historical literature). In a similar vein, Flournoy (1893) notes that the personification of his Mme. L. for Sunday had the face of her father, a pastor. By suggesting this is no coincidence, Flournoy implies that Mme. L.'s OLP is influenced by the learned knowledge not only of her father's features, but also of the religious connotation for Sunday in the Christian Church. If these observations are well founded, it would suggest that any biological predisposition for OLP could be tempered by experience, and this has been seen in many other variants of synesthesia. Ward and Simner (2003), for example, show that the taste concurrents experienced in lexical-gustatory synesthesia are significantly related to childhood diet, such that foods that were most often eaten are more likely to occur as synesthetic tastes. Equally, Simner et al. (2005) showed that graphemecolor synesthetes tend to rely on learned frequency knowledge in the pairings of their letters and colors (i.e., pairing high-frequency graphemes with highfrequency colors). We propose a similar account herethat a (possibly inherited) disposition for OLP is shaped by the experiences of the subject.

\section{Conclusion}

In summary, we have shown that the tendency to attribute animate qualities to linguistic ordinal sequences tends to co-occur with other variants of synesthesia and shares their characteristics of consistency, automaticity, and letter to word transference. Hence, OLP may arise as a result of similar cognitive mechanisms, some of which may be influenced by experience. We suggest, too, that OLP may stem from similar neurodevelopmental events, and data from future imaging studies may provide greater evidence for this hypothesis. Finally, our survey found a prevalence of OLP (gender and/or personality) of $1.4 \%$, and although our sample size was relatively small $(n=$ 219) our study represents the first estimates of OLP's prevalence that uses random sampling and verification with objective tests of genuineness.

\section{Acknowledgments}

This research was supported by J. S.'s Leverhulme Early Career Fellowship (40166).

The authors thank participant A.P. for her generous input, and Ed Hubbard for his helpful discussion about cross talk theories. We also thank the following for their help in data collection: Carolyn Black, Catherine Carew Hunt, Jenny Harold, Julia MacFarquhar, Louise Monro, Helen Moore, Jennifer Park, Sam Rothberg, Eleanor Smallwood, Robert Sproul-Cran, and Laura Tully.

Reprint requests should be sent to Julia Simner, Psychology, PPLS, University of Edinburgh, 7 George Square, Edinburgh EH8 9JZ, UK, or via e-mail: j.simner@ed.ac.uk.

\section{Note}

1. Of the 21 high scorers after 5 weeks only, 5 had consistency in grapheme-colors only, 6 in OLP only, and 10 in both. A year later, 2 in the color-only group remained consistent, 2 in the OLP-only group, and 1 in the group for both. Additionally, 4 members of this latter group remained consistent only for color, and 1 remained consistent but only for OLP.

\section{REFERENCES}

Baron-Cohen, S., Burt, L., Smith-Laittan, F., Harrison, J., \& Bolton, P. (1996). Synaesthesia: Prevalence and familiarity. Perception, 25, 1073-1079.

Baron-Cohen, S., Wyke, M. A., \& Binnie, C. (1987). Hearing words and seeing colours: An experimental investigation of a case of synaesthesia. Perception, 16, 761-767.

Beeli, G., Esslen, E., \& Jäncke, L. (2005). When coloured sounds taste sweet, Nature, 434, 38.

Calkins, M. W. (1893). A statistical study of pseudochromesthesia and of mental-forms. American Journal of Psychology, 5, 439-464.

Chien, J. M., \& Fiez, J. A. (2001). Dissociation of verbal working memory: System components using a delayed serial recall task. Cerebral Cortex, 11, 1003-1014.

Cytowic, R. E. (2002). Synesthesia: A union of the senses. New York: Springer-Verlag.

Dixon, M. J., Smilek, D., Cudahy, C., \& Merikle, P. M. (2000). Five plus two equals yellow. Mental arithmetic in people with synaesthesia is not coloured by visual experience. Nature, 406, 365.

Dixon, M. J., Smilek, D., \& Merikle, P. M. (2004). Not all synaesthetes are created equal: Projector versus 
associator synaesthetes. Cognitive, Affective, \& Behavioral Neuroscience, 4, 335-343.

Flournoy, T. (1893). Des phenomenes de synopsie. Paris: Felix Alcan.

Grossenbacher, P. G. (1997). Perception and sensory information in synaesthetic experience. In S. Baron-Cohen \& J. E. Harrison (Eds.), Synaesthesia: Classic and contemporary readings (pp. 148-172). Oxford: Blackwell.

Grossenbacher, P. G., \& Lovelace, C. T. (2001). Mechanisms of synaesthesia: Cognitive and physiological constraints. Trends in Cognitive Sciences, 5, 36-41.

Guthrie, A. (1993). Faces in the clouds. Oxford: Oxford University Press.

Heberlein, A. S., \& Saxe, R. R. (2005). Dissociation between emotion and personality judgements: Convergent evidence from functional neuroimaging. Neuroimage, 28, 770-777.

Hubbard, E. M., Arman, A. C., Ramachandran, V. S., \& Boynton, G. M. (2005). Individual differences among grapheme-color synesthetes: Brain-behavior correlations. Neuron, 45, 975-985.

Hubbard, E. M., \& Ramachandran, V. S. (2005). Neurocognitive mechanisms of synesthesia. Neuron, 48, 509-520.

Looft, W. R., \& Bartz, W. H. (1969). Animism revived. Psychological Bulletin, 71, 1-19.

Marakas, G. M., Johnson, R. D., \& Palmer, J. W. (2000). A theoretical model of differential social attributions towards computer technology: When the metaphor becomes the model. International Journal of Human-Computer Studies, 52, 719-750.

Maurer, D. (1997). Neonatal synaesthesia: Implications for the processing of speech and faces. In S. Baron-Cohen \& J. E. Harrison (Eds.), Synaesthesia: Classic and contemporary readings (pp. 224-242). Oxford: Blackwell.

Mauer, D., \& Mondloch, C. J. (2005). Neonatal synesthesia: A re-evaluation. In L. C. Robertson \& N. Sagiv (Eds.), Synesthesia: Perspectives from cognitive neuroscience (pp. 193-213). Oxford: Oxford University Press.

Mills, C. B., Boteler, E. H., \& Oliver, G. K. (1999). Digit synaesthesia: A case study using a Stroop-type test. Cognitive Neuropsychology, 16, 181-191.

Morris, P., Fidler, M., \& Costall, A. (2000). Beyond anecdotes: An empirical study of "anthropomorphism." Society and Animals, 8, 151-165.

Nunn, J. A., Gregory, L. J., Brammer, M., Williams, S. C. R., Parslow, D. M., Morgan, M. J., et al. (2002). Nature Neuroscience, 5, 371-375.

Palmeri, T. J., Blake, R., Marois, R., Flanery, M. A., \& Whetsell, W. (2002). The perceptual reality of synesthetic colors, Proceedings of the National Academy of Sciences, U.S.A., 99, 4127-4131.

Patrick, G. T. W. (1893, February). Number forms. Popular Science Monthly, 508-510.

Piaget, J. (1929). The child's conception of the world. New York: Harcourt Brace.

Piaget, J. (1933). The child's conception of physical causality. London: Keegan Paul.
Poldrack, R. A., Wagner, A. D., Prull, M. A., Desmond, J. E., Glover, G. H., \& Gabrielli, J. D. (1999). Functional specialisation for semantic and phonological processing in the left inferior prefrontal cortex. Neuroimage, 10, 15-35.

Psychological Software Tools. (2002). E-Prime. www.pstnet.com.

Ramachandran, V. S., \& Hubbard, E. M. (2001a). Psychophysical investigations into the neural basis of synaesthesia. Proceedings of the Royal Society of London B, 268, 979-983.

Ramachandran, V. S., \& Hubbard, E. M. (2001b). Synaesthesia-A window into perception, thought and language. Journal of Consciousness Studies, 8, 3-34.

Rich, A. N., Bradshaw, J. L., \& Mattingley, J. B. (2005). A systematic large-scale study of synaesthesia: Implications for the role of early experience in lexical-colour associations. Cognition, 98, 53-84.

Sagiv, N. (2005). Synesthesia in perspective. In L. C. Robertson \& N. Sagiv (Eds.), Synesthesia: Perspectives from cognitive neuroscience (pp. 3-10). Oxford: Oxford University Press.

Sagiv, N., Simner, J., Collins, J., Butterworth, B., \& Ward, J. (2006). What is the relationship between synaesthesia and visuo-spatial number forms? Cognition, 101, 114-128.

Simner, J., Glover, L., \& Mowat, A. (2006). Linguistic mechanisms of grapheme-colour synaesthesia. Cortex, 42, 281-289.

Simner, J., \& Hubbard, E. M. (2006). Variants of synaesthesia interact in cognitive tasks: Evidence for implicit associations and late connectivity in cross-talk theories. Neuroscience, 143, 805-814.

Simner, J., Mulvenna, C., Sagiv, N., Tsakanikos, E., Witherby, S. A., Fraser, C., et al. (2006). Synaesthesia: The prevalence of atypical cross-modal experiences. Perception, 35, 1024-1033.

Simner, J., Ward, J., Lanz, M., Jansari, A., Noonan, K., Glover, L., et al. (2005). Non-random associations of graphemes to colours in synaesthetic and non-synaesthetic populations. Cognitive Neuropsychology, 22, 1069-1085.

Stroop, J. R. (1935). Studies of interference in serial verbal reactions. Journal of Experimental Psychology, 18, 643-662.

Tyler, C. W. (2005). Varieties of synesthetic experience. In L. C. Robertson \& N. Sagiv (Eds.), Synesthesia: Perspectives from cognitive neuroscience (pp. 34-46). Oxford: Oxford University Press.

Ward, J., Huckstep, B., \& Tsakanikos, E. (2006). Sound-colour synaesthesia: To what extent does it use cross-modal mechanisms common to us all? Cortex, 42, 264-280.

Ward, J., \& Simner, J. (2003). Lexical-gustatory synaesthesia: Linguistic and conceptual factors. Cognition, 89, 237-261.

Ward, J., \& Simner, J. (2005). Is synaesthesia an x-linked dominant trait with lethality in males? Perception, 34, 611-623.

Ward, J., Simner, J., \& Auyeung, V. (2005). A comparison of lexical-gustatory and grapheme-colour synaesthesia. Cognitive Neuropsychology, 22, 28-41. 
Copyright of Journal of Cognitive Neuroscience is the property of MIT Press and its content may not be copied or emailed to multiple sites or posted to a listserv without the copyright holder's express written permission. However, users may print, download, or email articles for individual use. 